\section{Gastro-Highlights 2000}

Zusammenfassung der Referate anlässlich des gastroenterologischen Symposiums am Universitätsspital Zürich, 1. Juli 2000. Leitung: Professor Michael Fried

E. L. Renner, T. Roesch, P. Bauerfeind, W. Schwizer, H.-P. Wirth, C. Beglinger, $M$. Fried

Wie in jedem Jahr fanden auch in diesem Sommer die Gastro-Highlights in Zürich statt. Ziel dieser nun schon traditionellen Fortbildungsveranstaltung ist es, die während der jeweils vergangenen zwölf Monate gewonnenen, an der renommierten «Digestive Disease Week" (DDW) kurz zuvor vorgestellten, wichtigsten und praxisrelevanten Erkenntnisse aus dem Fachgebiet der Gastroenterologie/Hepatologie an praktizierende Gastroenterologen und Internisten weiterzugeben.

\section{Hepatologie}

\section{NASH-Syndrom}

Während der Zusammenhang zwischen Alkoholabusus und Leberzirrhose seit langem bekannt ist, sind die Daten zur Progredienz der nicht-alkoholischen Steatose/Steatohepatitis - NASH-Syndrom - viel unsicherer. P. Angulo et al. (1999) dokumentierten bei $20 \%$ von 144 Patienten mit bioptisch gesichertem NASH-Syndrom eine höhergradige Fibrose und bei 17\% eine voll ausgebildete Zirrhose [1]. Fazit: In 10 bis $20 \%$ der NASH-Fälle ist mit einer progredienten Fibrose bis hin zur Zirrhose zu rechnen.

Prädiktive Faktoren für eine Progression der nichtalkoholischen Steatose/Steatohepatitis zur Fibrose oder weiter zur Zirrhose sind bisher ungenügend definiert. Alter über 45 Jahre, starkes Übergewicht (Body Mass Index über $32 \mathrm{~kg} / \mathrm{m}^{2}$ ), Diabetes mellitus sowie ein AST/ALT-Verhältnis über 1 scheinen gemäss der Arbeit von Angulo et al. dazuzugehören [1].

\section{Virale Hepatitiden}

Eine antivirale Therapie mit Lamivudin konnte bei Patienten mit dekompensierter Hepatitis-B-Zirrhose nicht nur die HBV-Replikation hemmen, sondern führte auch zu einer Verbesserung der Leberfunktion (Child-Pugh-Score) [2]. Dies dürfte sich günstig auf das Überleben respektive die Notwendigkeit einer

Korrespondenz:

Prof. Dr. med. Michael Fried

Universitätsspital

DIM, Gastroenterologie

Rämistrasse 100

CH-8091 Zürich

E-mail: michael.fried@dim.usz.ch
Lebertransplantation auswirken. Als Pferdefuss der Monotherapie mit Lamivudin trat aber auch in dieser Studie innert weniger Monate in drei von 35 Fällen eine Lamivudin-Resistenz auf. Wie sich dies mittelfristig auf den Krankheitsverlauf bei (dekompensierter) Hepatitis-B-Zirrhose auswirkt, ist noch zu wenig bekannt.

In der Therapie der chronischen Hepatitis $\mathrm{C}$ wird neu PEG-(Polyäthylenglykol-)Interferon geprüft. Dies ist eine Art Depotinterferon, das nur noch einmal pro Woche verabreicht werden muss, aber dennoch konstante Plasmakonzentrationen erreichen lässt. Nach den bisher vorliegenden Studien scheint die Wirkung von PEG-Interferon alleine derjenigen der Kombination von herkömmlichem Interferon alpha plus Ribavirin vergleichbar. Bei allen Genotypen wirkt PEGInterferon besser als Interferon alpha [3, 4]. Auch ist PEG-Interferon wirksam bei Hepatitis-C-Patienten mit fortgeschrittener Fibrose respektive Zirrhose [5]. Die Verträglichkeit entspricht der von Interferon alpha [3, 4].

\section{Cholestatische Lebererkrankungen}

Bei der primär biliären Zirrhose (PBZ) handelt es sich um eine cholestatische Autoimmunkrankheit der Leber, die sich - unbehandelt - zur Leberzirrhose entwickelt. Einzige etablierte Therapie ist Ursodeoxycholsäure, mit der die Progression etwas verzögert, die Krankheit aber nicht geheilt werden kann. Bei ungenügendem Ansprechen wurden immer wieder Immunsuppressiva bzw. Steroide versucht, wobei es naheliegt, Substanzen mit hohem First-pass-Effekt und geringer systemischer Wirkung zu verwenden. Ein solches Glukokortikoid ist Budesonid, das zu 90\% in der Leber metabolisiert wird und somit nur noch $\mathrm{zu}$ 10\% in die Zirkulation gelangt. In Kombination mit Ursodeoxycholsäure hat sich Budesonid in einer Studie mit insgesamt 40 Patienten bei PBZ als wirksam erwiesen. Leberhistologie und verschiedene Laborparameter verbesserten sich im Vergleich zur Gabe von Ursodeoxycholsäure plus Plazebo signifikant [6]. Leider sind aber die Effekte in einer anderen - allerdings anders konzipierten - Studie [7] nicht so günstig ausgefallen, und Budesonid führte in letzterer, nicht aber in ersterer doch zu einer Abnahme der Knochendichte.

Zusätzliche Studien zur Kombination von Budesonid und Ursodeoxycholsäure sind folglich nötig, zumal Daten zu sogenannt harten Endpunkten (Überleben, Notwendigkeit der Transplantation) fehlen.

\section{Hereditäre Lebererkrankungen}

Wurde ein Zusammenhang zwischen Insulinresistenz bzw. Diabetes mellitus und Eisenüberladung (ausserhalb einer hereditären Hämochromatose) schon länger vermutet, konnte dieser jetzt erstmals belegt werden. In der Analyse von Mendler et al. zeigte sich bei mehr als der Hälfte der 161 Patienten mit bioptisch gesicherter Eisenüberladung der Leber, welche gemäss genetischen Untersuchungen keine hereditäre Hämochromatose aufweisen, ein Insulinresistenzsyndrom mit abnormer Glukosetoleranz, Hyperlipid- 
ämie und Adipositas (Body Mass Index $>25 \mathrm{~kg} / \mathrm{m}^{2}$ ) [8]. Möglicherweise wurde hier eine neue Krankheit definiert, deren Ursachen und Pathogenese ebenso wie der Verlauf allerdings noch unklar sind.

\section{Lebertumoren}

Ist bei Patienten mit Leberzirrhose (Child A) eine Transplantation angezeigt? Die Studie von J. M. Liovet und Mitarbeitern zeigt sehr schön, dass das (rezidivfreie) mittelfristige Überleben bei kleinen $\mathrm{Tu}$ moren nach Transplantation deutlich besser ist als nach Resektion, sofern die Wartezeiten auf das Transplantat kurz genug sind [9]. Gerade letzteres wird aber weltweit beim zunehmenden Auseinanderklaffen zwischen Bedarf und Verfügbarkeit von Spenderorganen ein immer grösseres Problem. Hier sind unter anderem Strategien gefragt, mit denen das Tumorwachstum bei den Patienten auf der Warteliste wirkungsvoll eingedämmt werden kann.

\section{Endoskopie}

\section{Endoskopische Histologie}

Deutliche Fortschritte sind in der endoskopischen Bildgebung zu verzeichnen. In Zukunft ist es denkbar, Bilder von einzelnen Zellen der Mukosa zu gewinnen, die histologischen Schnitten nahekommen [10]. Vor allem in Publikationen aus Japan sind mittels der "Laser Scanning Confocal Microscopy" gewonnene Bilder zu finden, in denen einzelne Zellen und Zellkerne erkennbar sind. Eine weitere neue Methode ist die optische Kohärenztomographie, bei der die (optische) Inhomogenität von Geweben genutzt wird. Die resultierenden Bilder sind ähnlich wie beim Ultraschall. Allerdings wird hier die Reflexion von Infrarotlicht anstelle des Schalls gemessen [11, 12].

Aber auch in der Routinediagnostik tut sich etwas, speziell in der Primärdiagnostik und Verlaufskontrolle des Barrett-Ösophagus. Noch immer nicht gelöst ist allerdings die Frage: Wer soll am ösophagogastralen Übergang biopsiert werden? Auch scheint die Diskussion unter Pathologen noch nicht abgeschlossen; die Bedeutung des intestinalen Epithels am ösophagogastralen Übergang (Kardia-Metaplasie? Barrett-Schleimhaut?) ist noch nicht abschliessend geklärt - ein solches Epithel ist bei 15 bis 30\% der Patienten mit Refluxkrankheit zu finden [13, 14]. Nur selten haben die Patienten eine völlig glatte Z-Linie, die keinen Verdacht auf einen kurzen Barrett aufkommen lässt. Bis diese Frage geklärt ist, sollen eine irreguläre Z-Linie und insbesondere kurze Zungen biopsiert werden.

Tonelotto und Mitarbeiter versuchten, sich die Diagnostik mit Hilfe der Vergrösserungsendoskopie zur Differenzierung zwischen Kardia- und BarrettSchleimhaut zu erleichtern. Bei einem "Magen-Typ» (Typ-I-Aussehen) fanden sie in keinem ihrer insgesamt 44 Fälle eine intestinale Metaplasie; hingegen bei 85\% der Patienten mit "Intestinal-Typ» (Typ-IIAussehen) in der Vergrösserungsendoskopie [15].
Wird ein «Barrett» nachgewiesen - wie soll der Patient überwacht werden? Die vorliegenden Arbeiten zur Bedeutung der Methylenblaufärbung für die Erkennung einer hochgradigen Dysplasie bzw. eines Frühkarzinoms sind uneinheitlich, die Resultate schlecht vergleichbar. Am günstigsten ist es wahrscheinlich für den Endoskopiker, sich mit dem unterschiedlichen Aussehen «normaler» und (prä-) maligne veränderter Barrett-Schleimhaut vertraut zu machen und genau hinzuschauen; endoskopische BarrettÜberwachungen sind deshalb zeitaufwendige Untersuchungen.

\section{Endoskopische Refluxtherapie}

Mit relativ viel Lärm wurden an der DDW in San Diego drei Techniken vorgestellt. Eine davon - die endoskopische Nähmaschine nach Paul Swain - erscheint nicht übermässig elegant. In einer amerikanischen Multizenterstudie konnten zwar die Symptome einigermassen, nicht aber der Refluxscore mit dieser Methode wesentlich verbessert werden [16]. Der Erfinder der Methode selber kann hingegen gute Ergebnisse vorweisen [17].

Solidere Daten können Triadafilopoulos und Mitarbeiter für ihre "Stretta procedure" präsentieren eine Form der Radiofrequenzenergieapplikation in die Wand des ösophagogastralen Übergangs. Nach einer Beobachtungszeit von sechs Monaten haben die Patienten mit Reflux und Ösophagitis (Grad I und II) deutlich weniger Sodbrennen, eine bessere Lebensqualität und eine verkürzte Zeitphase mit einem sauren $\mathrm{pH}-$ Wert $(<4)[18]$.

\section{MRCP}

Der klinisch sinnvolle Einsatz der MRCP ist bisher kaum zuverlässig evaluiert, der tatsächliche Nutzen noch nicht erfassbar. Das für diese neue radiologischendoskopische Methode geeignete Patientengut ist deshalb auch nur ungenau definiert. Geeignet erscheint die MRCP nach eigenen Ergebnissen jedoch zur Diagnose von Pankreas-Erkrankungen. Hier erreicht die Spezifität 100\%, die Sensitivität im Mittel $90 \%$ [19].

Ganz sicher derzeit nicht geeignet ist die MRCP zum Screening bei Patienten mit rechtsseitigen Oberbauchbeschwerden oder leicht erhöhten Cholestaseoder Pankreaswerten. Ein möglicher Einsatzbereich ist gegeben bei unklarer Indikation für eine ERCP, eventuell vor einer therapeutischen ERCP. Insgesamt jedoch müssen die Indikationen für eine MRCP noch evaluiert und definiert werden.

\section{Virtuelle Koloskopie}

Die virtuelle Koloskopie macht mit phantastischen Bildern von sich reden. Unklar ist jedoch noch, inwieweit sie sich zum Screening bei Patienten mit Polypen eignet, und wann sie kosteneffizient wird. In der Arbeit von Fenlon und Mitarbeitern zeigt sich, dass die Treffsicherheit der virtuellen Koloskopie um so schlechter ist, je kleiner die Polypen sind [20]. Rund 20\% der Befunde sind nach den bisherigen Erfahrungen falsch positiv. 


\section{Kolontumore und Funktionsstörungen des Kolons}

\section{Kolonkarzinom-Screening}

Screening auf Kolonkarzinom ist ein Thema, das Patienten und Hausärzte mit am meisten interessiert. Vier Methoden sind dazu verfügbar, deren Durchführung ratsam ist: Hämokkult-Test auf okkultes Blut im Stuhl, Sigmoidoskopie oder die Kombination von beidem, Barium-Kontrasteinlauf und Koloskopie.

\section{Hämokkult}

Theoretisch betrachtet ist der Test auf okkultes Blut eine - aufgrund der ungenügenden Sensitivität schlechte Screeningmethode, der Gastroenterologen berechtigterweise skeptisch gegenüberstehen. Interessanterweise jedoch lässt sich mit dieser Form des Screenings die Mortalität an kolorektalen Karzinomen um ansehnliche 33\% in 18 Jahren reduzieren, wie Mandel et al. zeigen [21] und damit frühere Ergebnisse bestätigen. Der Einsatz des Hämokkult-Tests ist also besser als gar kein Screening. Entscheidend ist, dass der Test nur bei asymptomatischen Personen angewandt wird. Sobald Patienten auf Kolonkarzinom verdächtige Symptome haben oder einer Risikopopulation angehören, darf der Test wegen seiner geringen Sensitivität nicht verwendet werden.

\section{Sigmoidoskopie}

Eine Arbeitsgruppe konnte anhand einer randomisierten Untersuchung an 328 Patienten zeigen, dass Krankenschwestern ebenso gut sigmoidoskopieren wie Ärzte [22]. Ärzte verpassten 20\% und Schwestern 21\% der Polypen.

\section{Barium-Kontrasteinlauf und Koloskopie}

Einer Analyse von Winawer et al. zufolge ist der in Europa ohnehin selten durchgeführte Barium-Doppel-Kontrasteinlauf der Koloskopie deutlich unterlegen [23] und im klinischen Alltag kaum nützlich. Die Sensitivität erreicht in einer Subgruppenanalyse der amerikanischen National Polyp Study $(\mathrm{n}=580)-$ je nach Polypgrösse - lediglich 30 bis $50 \%(<0,5 />1 \mathrm{~cm})$, ein ernüchterndes Ergebnis [23].

\section{Hereditäres nicht-polypöses Kolonkarzinom (HNPCC)}

$10 \%$ aller kolorektalen Karzinome gehören in diese Gruppe. Die zur Definition dienenden AmsterdamKriterien wurden 1999 revidiert. Zum HNPCC zählen Patienten auch dann, wenn mindestens drei Verwandte mit einem kolorektalen Karzinom oder einem Karzinom des Endometriums, Dünndarms, Ureters oder Nierenbeckens bestehen. Die Konsequenz: engmaschige koloskopische, gynäkologische und urologische Überwachung der Risikopatienten zwecks Entdeckung auch der assoziierten Karzinome.

Durch regelmässiges Screening alle drei Jahre lassen sich, wie eine finnische Arbeitsgruppe an 252 Patienten belegt, sowohl Inzidenz als auch Mortalität des HNPCC massiv senken [24]. Die Beobachtungszeit betrug in dieser Studie 15 Jahre.
In der Schweiz wird das HNPCC zu selten erfasst, weil zumeist nicht früh genug daran gedacht wird. Bei Risikopatienten kann eine genetische Abklärung durchgeführt werden. Bei Personen mit genetischer Disposition empfiehlt sich ab einem Alter von 25 Jahren eine Koloskopie alle zwei Jahre. Eine Chemoprävention mit nicht-steroidalen Antirheumatika (NSAID) ist aller Wahrscheinlichkeit nach in diesen Fällen nicht sinnvoll.

\section{Kolonkarzinom-Prävention}

Eine ganze Reihe von Nahrungsmitteln, Nahrungsbestandteilen und Medikamenten wird seit Jahren regelmässig in Zusammenhang mit dem in der Bevölkerung so häufigen Kolonkarzinom genannt. Prospektive Studien sind rar; die wenigen prospektiven Untersuchungen zeigten meist keine Wirkung. Noch immer als wichtig wird der Konsum faserreicher Kost betrachtet; auch wenn die neuen Studien über eine Dauer von drei bis vier Jahren keine Inzidenzsenkung belegen $[25,26]$.

Sinha und Mitarbeiter bestätigen in einer prospektiven Fall-Kontroll-Studie einmal mehr die Annahme, dass Fleischkonsum (vor allem durchgebratenes rotes Fleisch) einen Risikofaktor für das Kolonkarzinom darstellt [27].

Kalzium hat sich letztlich als der einzige Faktor herauskristallisiert, der sich prospektiv als präventiv wirksam erwiesen hat. Die Arbeitsgruppe um Baron konnte in ihrer vierjährigen plazebokontrollierten Studie mit 930 Adenom-Patienten (Sekundärprävention, nach Therapie) durch Verabreichung von 1200 mg Kalzium täglich eine signifikante Polypenreduktion um 15\% erzielen $(p=0,03)$ [28].

Den NSAID wird seit längerem eine präventive Wirkung zugesprochen, obwohl prospektive Studien das bisher nicht belegten. Tierexperimente deuten darauf hin, dass der Effekt überwiegend COX-2-abhängig ist. Vor diesem Hintergrund wurde mit Spannung die erste, im Juni publizierte Studie mit einem der neuen COX-2-Hemmer abgewartet. Die Resultate sind jedoch enttäuschend. Zwar konnte unter Patienten mit familiärer Polyposis durch Celecoxib (100 bzw. $400 \mathrm{mg}$ zweimal täglich) im vorgegebenen Zeitraum dosisabhängig das Karzinomrisiko verglichen mit Plazebo gesenkt werden (um 12 respektive $28 \%$ ), die Streubreite war jedoch enorm [29].

\section{Colon irritabile}

Die Behandlung des irritablen Kolons erweist sich erfahrungsgemäss als relativ undankbar. Mit Alosetron steht ein neuer 5-HT3-Antagonist zur Verfügung, der sicher und wirksam zu sein scheint. Das zumindest ist die Schlussfolgerung der Arbeitsgruppe um Camilleri, die in einer plazebokontrollierten Studie den Wirkstoff während zwölf Wochen an insgesamt 647 Frauen mit Colon irritabile (70\% litten vor allem unter Diarrhöe) geprüft haben [30]. 41\% der Patientinnen hatten unter Alosetron weniger Schmerzen, verglichen mit 29\% unter Plazebo. Zu denken gibt die hohe Drop-out-Rate $(\mathrm{n}=79$ unter Verum, $\mathrm{n}=53$ unter Plazebo). 
Alternativen bietet die Naturmedizin in Form einer chinesischen Kräuterarznei (Aristolochia fangchi) an, die eine dem Alosetron fast vergleichbare symptomatische Wirkung erzielt [31]. Die Sicherheit der Naturmittel ist unklar.

\section{Refluxkrankheit}

Hiatushernie

Welche Bedeutung hat die Hiatushernie? Der Zusammenhang mit der Refluxkrankheit ist nicht zu übersehen - 50 bis 94\% der Refluxpatienten haben diesen Befund - und es stellt sich die Frage, ob sie ein Faktor in der Pathogenese ist oder lediglich eine Koinzidenz. Bei Patienten mit Hiatushernie treten die meisten Refluxepisoden beim Schlucken auf. Peter J. Kahrilas beobachtete nun eine lineare Assoziation zwischen der Grösse einer Hernie und der Häufigkeit transienter Sphinkterrelaxationen, die bekanntlich zu vermehrtem Reflux führen [32]. Er bestätigt somit die ursächliche Rolle der spontanen Relaxation des unteren Sphinkters, die bei Gesunden etwa zweieinhalbmal pro Stunde stattfindet, bei Refluxpatienten sechs- bis siebenmal und bei Hernienpatienten etwa zwölfmal pro Stunde. Die Hiatushernie ist somit ein Faktor in der Pathogenese der Refluxkrankheit, jedoch nicht alleine dafür verantwortlich.

Langzeitbehandlung mit Protonenpumpenblockern (PPI)

Wie wirkt sich eine Langzeitbehandlung mit PPI auf die Magenschleimhaut aus? Diese Frage ist, neben der Wirksamkeit, Gegenstand der Studie von E. C. Klinkenberg-Knol, in der 230 Patienten mit Refluxösophagitis unter Omeprazol-Therapie über 6,5 Jahre beobachtet und regelmässig alle sechs Monate kontrolliert wurden [33]. Die Studie zeigt ein weiteres Mal die zuverlässige Wirkung von Omeprazol (20 mg täglich) auf, was sich an der geringen Rezidivrate ablesen lässt: 1 Fall pro 8,5 Behandlungsjahre bei Helicobacter-pylori-(Hp-)negativen Patienten sowie 1 Fall pro 10 Behandlungsjahre bei Hp-positiven Patienten. Bei allen Patienten mit einem Rückfall heilte die Refluxösophagitis unter Omeprazol aus, bei knapp der Hälfte (40\%) war eine erhöhte Dosierung dafür erforderlich.

Unter der Omeprazol-Therapie gab es keine Komplikationen, und es wurden weder Dysplasien noch Neoplasien der Magenmukosa dokumentiert [33]. Lars Lundell kommt in seiner dreijährigen Studie mit 310 Refluxpatienten zum Schluss, dass eine langzeitige Säuresuppression bei Hp-positiven Patienten nicht zu einer Atrophie der Magenschleimhaut führt und nicht zum Auftreten einer intestinalen Metaplasie [34].

\section{Barrett-Ösophagus}

Ist der insgesamt häufigere Kurzsegment-Barrett $(<3 \mathrm{~cm})$ bezüglich des Adenokarzinomrisikos weniger gefährlich als der sogenannt klassische Barrett (>3 cm)? Eine Frage, die Auswirkungen auf das Screening hat, ist doch das Adenokarzinomrisiko bei
Patienten mit Barrett-Ösophagus 30- bis 125fach erhöht. R. Rudolph in Seattle findet in seiner Studie mit insgesamt 309 Patienten keinen Unterschied [35]. Danach ist die Progression zum Adenokarzinom in etwa die gleiche für den Kurzsegment- wie für den Langsegment-Barrett.

Sharma et al. zeigten auch, dass das Dysplasierisiko bei Patienten mit intestinaler Metaplasie im Kurzsegment-Barrett viel höher ist als bei einer intestinalen Metaplasie der Magenkardia [36].

Lässt sich die Dysplasie des Ösophagusepithels durch Säuresuppression verhindern? Die Antwort lautet wahrscheinlich ja, obwohl diese Frage noch durch keine kontrollierte Studie untersucht wurde. Es gilt jedoch zu berücksichtigen, dass die Symptomfreiheit unter säurehemmender Behandlung kein zuverlässiges Kriterium zur Beurteilung der Therapiewirksamkeit bei Barrett-Patienten darstellt [37]. Bei 20 bis $40 \%$ der Patienten mit Barrett-Ösophagus ist - trotz Beschwerdefreiheit - die Säuresuppression unvollständig, so dass die Speiseröhre weiterhin einer erhöhten Säureexposition ausgesetzt ist [38, 39]. Das hat insofern Folgen, als Säure Zelldifferenzierung und Proliferation negativ beeinflusst. Eine pathologische Proliferation der Barrett-Mukosa hat eine verminderte Differenzierung der Epithelzellen zur Folge [38]. Säureexposition der Barrett-Mukosa begünstigt demnach die Entwicklung einer Dysplasie. Für die Praxis bedeutet das: Säuresuppression bei Patienten mit Barrett mittels 24-Stunden-pH-Metrie kontrollieren, Säuredurchbrüche (vor allem nächtliche) vermeiden.

\section{Antirefluxoperation}

Kontroverse Daten wurden von Bais et al. im Zusammenhang mit Komplikationen der laparotomischen versus laparoskopischen Antirefluxoperation publiziert. Im Vergleich zur Laparotomie (offene Fundoplikatio) ist die Laparoskopie (laparoskopische Fundoplikatio) mit einer höheren Rate an intra- und perioperativen Komplikationen behaftet [40]. J. E. Bais beziffert das relative Risiko der Laparoskopie für Dysphagie, Reflux und Herniation mit 8,8. Auch in der Übersicht von T. K. Rantanen in Finnland (er analysierte 5502 Antirefluxoperationen) schneidet die Laparoskopie schlechter ab als die Laparotomie: Tödliche und lebensbedrohliche Komplikationen waren nach Laparoskopie signifikant häufiger [41].

Dass Nausea auch eine Folge der Refluxkrankheit sein kann, wird einmal mehr durch die Arbeit von Hui bestätigt. Er berichtet über gute Erfolge der laparoskopischen Antirefluxoperation bei den eher seltenen Refluxpatienten mit chronischer Nausea. Bei seinen Patienten waren knapp zwei Drittel der Fälle nach der Antirefluxoperation geheilt [42].

\section{Dyspepsie, Ulkuserkrankungen und \\ Helicobacter pylori}

\section{Dyspepsie}

Wann soll bei einem Patienten mit Dyspepsie eine spezifische Therapie eingeleitet werden? $\mathrm{Zu}$ dieser 
Frage stellt Nick Talley einen neuen Algorithmus mit leicht angehobener Altersgrenze vor [43]. Danach sollte bei einem unter 50jährigen Patienten mit seit vier Wochen bestehender Dyspepsie auf Helicobacter pylori (Hp) getestet werden. Ist der Test positiv, wird eine Eradikation vorgeschlagen; ist er negativ, eine empirische Therapie; spricht der Patient darauf nur ungenügend an, sollte endoskopiert werden.

Bei einem Patienten über 50 oder bei Vorliegen von Alarmsymptomen oder Einnahme von NSAID sollte stets endoskopiert werden, wenn die Dyspepsie über vier Wochen persistiert. Bei nachgewiesener struktureller Erkrankung ist eine spezifische Therapie einzuleiten.

\section{Hp-Infektion}

Bekanntlich kann sich eine Hp-Infektion in einigen Situationen als vorteilhaft erweisen. So heilt eine Refluxösophagitis bei Hp-positiven Patienten unter PPI-Behandlung signifikant schneller ab als bei Hpnegativen [44].

Eine chronische Korpusgastritis - meistens durch Hp-Infektion bedingt - schützt interessanterweise vor einer Refluxösophagitis. Das ist das Resultat einer Analyse von El-Serag. Danach bestätigen sich Alter, männliches Geschlecht und Rauchen als Risikofaktoren für eine Refluxkrankheit, während die Korpusgastritis protektiv wirkt und das relative Risiko um $70 \%$ reduziert [45].

Keinen Einfluss hingegen hat offenbar die HpInfektion auf den Grad einer Ösophagitis, wie 0'Connor in einer Metaanalyse von 26 Studien mit insgesamt 2112 Patienten zeigt [46]. Die Prävalenz der Hp-Infektion ist allerdings höher bei Patienten ohne gastroösophagealen Reflux (GERD) als bei solchen mit Reflux. In 13 Fall-Kontroll-Studien beobachtet der Autor eine Hp-Infektion bei 39\% der Patienten mit Ösophagitis und GERD, verglichen mit 50\% der Patienten mit Ösophagitis ohne Reflux.

Den wichtigsten Aspekt der Rebound-Hypersekretion von Magensäure nach Absetzen einer PPITherapie zeigt die Studie von Gillen auf [47]. Dabei steigt die basale Säuresekretion bei Hp-negativen Personen stärker an als bei Hp-positiven. Demgegenüber zeigt die maximale Säuresekretion keinen Unterschied in bezug auf den Hp-Status.

\section{Ulkus, Ulkusblutung und NSAID}

Die Ulkusblutung stellt ein wichtiges Problem dar. Verschiedene Faktoren steigern bekanntlich das Risiko: das sind orale Antikoagulation (Odds-Ratio 7,8), Herzinsuffizienz (OR 5,9), Dyspepsie (OR 4,0), Status nach Ulkus (OR 3,8), Diabetes (OR 3,1), Kortikosteroide (OR 2,7) und Rauchen (OR 1,6) [48]. Ein sehr hohes Risiko ist gegeben, wenn Steroide und NSAID zusammen eingenommen werden (OR 9,0). Hp-positive Patienten unter NSAID haben ein höheres Blutungsrisiko als Hp-negative. Patienten mit Hp-Infektion plus Status nach Ulkusblutung besitzen das höchste Risiko (OR 9,74) [49].

Interessanterweise erhöhen die bei Depression eingesetzten selektiven Serotonin-Wiederaufnahme- hemmer (SSRI) das Risiko für eine obere gastrointestinale Blutung um den Faktor 3 [50], was angesichts der Verbreitung dieser Gruppe von Medikamenten von erheblicher praktischer Bedeutung ist.

Über die Hp-Therapie bei Patienten unter NSAID gibt es bisher keine klaren Empfehlungen. Am besten geeignet erscheint jedoch die prophylaktische Gabe von PPI (zum Beispiel Omeprazol $20 \mathrm{mg}$ ), falls die NSAID weiter eingenommen werden müssen.

Das Risiko für die Entstehung gastrointestinaler Ulzera unter nicht-steroidaler antirheumatischer Therapie ist bei den neuen spezifischen COX-2-Hemmern Celecoxib und Rofecoxib zwar reduziert [51, 52]. Ein Patient mit epigastrischen Schmerzen unter COX-2-Hemmer-Therapie kann aber sehr wohl ein Ulkus haben.

\section{Hp-Eradikation}

Drei wichtige neue potentielle Indikationsgebiete für eine Hp-Eradikation wurden in aktuellen Publikationen vorgestellt: die lymphozytäre, besonders die Korpus-Gastritis [53], das niedrig-maligne gastrische MALT-Lymphom auch mit tieferer Infiltration in die Magenwand [54] und die Eisenmangelanämie unklarer Ätiologie, die sich nach Eradikationstherapie zum Teil sehr gut zurückbildet und eine weitere Eisensubstitution unnötig werden lässt [55].

Dass mit der sogenannten Tripeltherapie die besten Eradikationsraten erzielt werden, wurde in einer Reihe von Studien erneut bestätigt [56]. Diese Raten im Bereich von 80 bis $90 \%$ kommen allerdings nur dank der hochwirksamen PPI zustande; wird der Säureblocker aus dem Therapieregime weggelassen, sinken die Eradikationsraten massiv ab [57]. Mit Esomeprazol steht ab dem nächsten Jahr ein neuer sehr potenter PPI zur Verfügung, der in der Eradikationstherapie als Bestandteil des Tripelregimes ersten Studien zufolge eine mindestens ebenso gute Keimeradikation bewirkt wie Omeprazol, nämlich zwischen 86 und 90\%; die Daten wurden an der DDW 2000 vorgestellt $[58,59]$.

Für die erfolgreiche PPI-Wirkung spielt der Patientengenotyp der PPI-Metabolisierung übrigens eine massgebliche Rolle. Nach neuesten Erkenntnissen schneiden sogenannte "poor metabolizer" mit langsamerem Abbau der PPI und folglich höheren Plasmakonzentrationen bei den PPI-haltigen Eradikationskuren besser ab als schnelle Metabolisierer. Interessant ist die Beobachtung, das die Infektion auch bei raschen Metabolisierern mittels einer PPI-Dosissteigerung eradiziert werden konnte, bei welchen die Ersttherapie zunächst versagt hatte [60]. Das bedeutet, dass den Wirtsdeterminanten in der Praxis mehr Beachtung geschenkt werden sollte als bis anhin.

Die Resistenz von Hp gegenüber Makroliden wie Clarithromycin scheint sich auf einem Niveau von rund 10\% einzupendeln, die gegenüber Nitroimidazolen wie Metronidazol hingegen steigt an [61].

Ein neu vorgeschlagenes Stufenschema trägt der gegenwärtigen Resistenzlage Rechnung. Erster Versuch: Tripeltherapie mit PPI, Makrolid (Clarithromycin) und Aminopenicillin (Amoxicillin); zweiter Ver- 
such mit Metronidazol anstelle von Clarithromycin. Es erscheint nicht ratsam, Clarithromycin und Metronidazol initial zu kombinieren. Schlägt auch der zweite Versuch fehl, sollte nach Antibiogramm behandelt werden, und zwar unter Einbezug von Hochdosis-PPI und Amoxicillin (modifiziert nach de Boer [62]).

$\mathrm{Zu}$ denken gibt derzeit ein anderer Aspekt im $\mathrm{Zu}$ sammenhang mit der Hp-Eradikation: die beträchtliche Induktion von Antibiotikaresistenzen in der Darmflora. Während resistente HP nach fehlgeschlagener Therapie im wesentlichen im Magen verbleiben, werden resistente Darmkeime noch während Wochen nach Beendigung der Therapie über den Stuhl ausgeschieden [63]. Entsprechend sollte die Notwendigkeit einer Keimausräumung auch aus diesen Gründen besonders eingehend geprüft werden. Die Frage, was im Zuge der chemotherapeutischen Hp-Eradikation mit der Mikroflora des Körpers passiert, wird zunehmend wichtiger.

Welches sind die möglichen Komplikationen einer Hp-Eradikation mittels Tripelregimes, bestehend aus PPI, Amoxicillin und Clarithromycin? Razavi berichtet in seiner kürzlich im Lancet publizierten Arbeit von pseudomembranöser Kolitis und Clostridiumdifficile-assoziierter reaktiver Arthritis [64]. Dabei handelt es sich zwar um eine seltene Komplikation: jedoch die pseudomembranöse Kolitis selbst wird als Komplikation eher zunehmen. Dies deshalb, weil in den aktuellen Therapieempfehlungen Metronidazol zunehmend durch Amoxicillin verdrängt wird.

\section{Chronisch entzündliche Darmerkrankungen}

\section{Morbus Crohn}

Vor allem in die Behandlung des aktiven Morbus Crohn ist mit der Einführung neuer Substanzen Bewegung gekommen. Derzeit sind in der Schweiz vier gegen Tumor-Nekrose-Faktor alpha (TNFalpha) gerichtete Arzneien verfügbar: Infliximab, Etanercept und CDP 571 als Antikörper sowie Thalidomid als TNFalpha-Rezeptorantagonist. Nur für Infliximab sind grössere klinische Studien vorliegend.

Mit dem Ziel der Definition des Stellenwertes der TNFalpha-Antikörper wurden verschiedene Studien durchgeführt und publiziert. Die Daten zeigen Remissionsraten zwischen 29 und 53\% [65-68]. Bei zwei Drittel der Behandelten wurde mit Infliximab eine deutliche Reduktion der Krankheitsaktivität erzielt, bei einem Drittel eine komplette Remission. Im Rahmen der Postmarketing-Überwachung von mehr als 50000 Crohn-Patienten wurden nach Infliximabbehandlung keine gravierenden Nebenwirkungen erfasst. Auch in einer aktuellen Studie zur Langzeittherapie über drei Jahre wurden nach Infliximabbehandlung bei den 770 Verum-Patienten keine Sicherheitsprobleme dokumentiert [69].

Insgesamt lässt sich also aufgrund der bisherigen Daten festhalten, dass die Sicherheit von Infliximab belegt ist. Die Inzidenz solider Tumoren und schwerer Infektionen ist nach dieser Therapie, verglichen mit Plazebo, nicht erhöht (4,8 versus 4,7\%); die gegenüber Plazebo erhöhte Lymphomhäufigkeit $(9,1$ versus 0\%) scheint im Zusammenhang mit der Grundkrankheit zu stehen; Infusionszwischenfälle sind relativ selten $(4,8 \%)[69,70]$.

Als akzeptierte Indikationen für eine Behandlung mit Infliximab gelten heute Patienten mit aktivem Morbus Crohn, die auf adäquate konventionelle Behandlung nicht ansprechen, sowie Patienten mit drainierenden Fisteln.

Dennoch verbleiben einige ungelöste Fragen $\mathrm{zu}$ dieser Therapieform. Das betrifft vor allem das Langzeitmanagement der Erkrankung. Die Patienten sprechen in der Regel dramatisch auf Infliximab an, können nach Absetzen jedoch ein Rezidiv entwickeln. Der natürliche Krankheitsverlauf wird also offenbar nicht beeinflusst. Bisher sind zudem keine spezifischen Faktoren bekannt, die eine Voraussage ermöglichen, wer auf diese Therapie anspricht.

Mit Thalidomid, einem potenten TNFalpha-Antagonisten, wurden bei Patienten mit aktivem Morbus Crohn in offenen, nicht plazebokontrollierten Studien gute Remissionsraten erzielt - 64 bis 70\% unter hoher Dosierung innerhalb von zwölf Wochen - die Problematik der Nebenwirkungen dieser Substanz sind jedoch weithin bekannt (Teratogenität). Der Einsatz von Thalidomid ist also sehr vorsichtig und wohlbegründet zu handhaben.

Eher enttäuschend sind die Resultate, die mit Mycophenolat Mofetil (MMF) bei Crohn-Patienten erzielt wurden [71, 72]. Das Fazit: MMF ist bei aktivem Morbus Crohn nicht in der Lage, eine Remission zu erzielen oder die Patienten in Remission zu halten. In Kombination mit Steroiden wirkt MMF nicht besser als die kombinierte Therapie mit Azathioprin plus Steroiden. Es besteht also - bei insgesamt noch ungenügender Datenlage - kein Anlass, MMF ausserhalb von klinischen Studien einzusetzen.

Lange Zeit von Gastroenterologen ignoriert, in der Rheumatologie hingegen gut bewährt, gewinnt Methotrexat in der Remissionserhaltung bei Morbus Crohn an Bedeutung. In einer plazebokontrollierten Doppelblindstudie von 40 Wochen Dauer mit insgesamt 76 Patienten wurde unter Methotrexat eine signifikant bessere Remission erzielt ( 65 versus 39\%) als unter Plazebo; auch die Steroidbedürftigkeit war unter dem Immunsuppressivum geringer als unter Plazebo (28 versus 39\%) [73].

Die potentiell unerwünschten Wirkungen von Methotrexat - Leberzirrhose und Pneumonitis - sind bekannt. Patienten mit Leberkrankheit oder mit Diabetes mellitus sollten nicht mit diesem Medikament behandelt werden.

Lassen sich die Therapieerfolge bei Crohn-Patienten durch den Einsatz von Wachstumshormonen verbessern? Einer Pilotstudie zufolge scheint das möglich. Die Krankheitsaktivität - gemessen am Abfall des CDAI-Scores - wird durch eine viermonatige Therapie (zusätzlich zur üblichen Medikation) deutlich gemindert [74].

Was bringt die postoperative Prophylaxe mit Mesalazin? Einer aktuellen prospektiven Studie, mit 
einer Dauer von 18 Monaten, zufolge hat diese Therapie (Mesalazin $4 \mathrm{~g}$ täglich), verglichen mit Plazebo, keinen Einfluss auf den postoperativen Verlauf bei Patienten mit Morbus Crohn [75].

\section{Colitis ulcerosa}

Die probiotische Behandlung der aktiven Colitis ulcerosa mit nicht-pathogenen E.-coli-Bakterien scheint eine Alternative für die Patienten darzustellen. In der ersten doppelblinden Vergleichsstudie mit insgesamt 116 Patienten ergaben sich jedenfalls keine signifikanten Unterschiede zwischen Mesalazin- und E.-coli-Applikation (jeweils in Kombination mit Steroiden und Gentamyzin) hinsichtlich der Remissionsraten und der Rezidive [76]. Die Verabreichung von E. coli (Nissle) bildet demnach einen interessanten Ansatz, erfordert jedoch noch zusätzliche Daten, zumal in der zitierten Studie Mesalazin in sehr niedriger Dosierung zum Einsatz kam.
Ein Problem der entzündlichen Darmerkrankungen ist das erhöhte Frakturrisiko. Wie eine in "Gut» publizierte Arbeit aufzeigt, sind davon vor allem Frauen betroffen, und das ganz speziell, wenn sie rauchen [77]. Steroide traten in dieser dänischen Analyse von insgesamt 998 Patienten und 1000 Kontrollpersonen nicht als Risikofaktor für Frakturen in Erscheinung.

\section{Krankheitskosten}

Daten aus den USA geben erste Hinweise auf die Kosten, die durch Morbus Crohn anfallen [78]. Danach werden 29\% der Behandlungskosten für 5-ASAPräparate aufgewendet. Der grösste Anteil fliesst in chirurgische Interventionen, wobei damit aber auch die längsten Remissionen erzielt werden. Generell erweisen sich Behandlungsstrategien als kostensenkend, die hohe Remissionsraten und anhaltende Remissionen bewirken.

\section{Literatur}

1 Angulo P, Keach JC, Batts KP, Lindor KD. Independent predictors of liver fibrosis in patients with nonalcoholic steatohepatitis. Hepatology 1999;30:1356-62.

2 Villeneuve JP, Condreay LD, Willems B, Pomier-Layrargues G, Fenyves D, Bilodeau M, et al. Lamivudine treatment for decompensated cirrhosis resulting from chronic hepatitis. Hepatology 2000;31:207-10.

3 Trepo C, Lindsay K, Niederau C, Shiffman M, Gordon S, Hoefs J, et al, for the Hepatitis Interventional Therapy Group. Pegylated interferon alfa-2b (PEG-Intron) monotherapy is superior to interferon alfa-2b (Intron A) for the treatment of chronic hepatitis C. J Hepatol 2000;32(Suppl 2):29.

4 Zeuzem S, Feinman SV, Rasenack J, Heathcote EJ, Lai MY Gane E, et al. Evaluation of the safety and efficacy of onceweekly PEG-interferon alfa-2a (PEGASYS) for chronic hepatitis C. A multinational randomized study. Hepatol 2000; 32(Suppl 2):29.

5 Heathcote J, Schiffman ML, Cooksley G, Dusheiko GM, Lee SS, Balart L, et al. Multinational evaluation of the efficacy and safety of once-weekly PEG-interferon alfa-2a in patients with chronic hepatitis $\mathrm{C}$ with compensated cirrhosis. Hepatology 1999;30:316A.

6 Leuschner M, Maier KP, Schlichting J, Strahl S, Herrmann G, Dahm HH, et al. Oral budesonide and ursodeoxycholic acid for treatment of primary biliary cirrhosis: results of a prospective double-blind trial. Gastroenterology 1999; 117:918-25.

7 Angulo P, Jorgensen RA, Keach JC, Dickson ER, Smith C, Lindor KD. Oral budesonide in the treatment of patients with primary biliary cirrhosis with a suboptimal response to ursodeoxycholic acid. Hepatology 2000;31:318-23.

8 Mendler MH, Turlin B, Moirand R, Jouanolle AM, Sapey T, Guyader D, et al. Insulin resistance-associated hepatic iron overload. Gastroenterology 1999;117:1155-63.

9 Llovet JM, Fuster J, Bruix J for the Barcelona Liver Clinic Cancer (BLCC) Group. Intention-to-treat analysis of surgical treatment for early hepatocellular carcinoma: resection versus transplantation. Hepatology 1999;30:1434-40.

10 Inoue H, Igari T, Nishikage T, Ami K, Yoshida T, Iwai T. A novel method of virtual histopathology using laser scanning confocal microscopy in-vitro with untreated fresh specimens from the gastrointestinal mucosa. Endoscopy 2000;32(6):439-43.

11 Jäckle S, Gladkova N, Feldchtein F, Terentieva A, Brand B, Gelikonov G, et al. In vivo endoscopic optical coherence tomography of the human gastrointestinal tract - toward optical biopsy. Endoscopy 2000;32:743-9.

12 Jäckle S, Gladkova N, Feldchtein F, Terentieva A, Brand B, Gelikonov G, et al. In vivo endoscopic optical coherence tomography of esophagitis, Barrett's esophagus, and adenocarcinoma of the esophagus. Endoscopy 2000;32:750-5.

13 Ott R, Roesch T, Mueblen J, Birkner B, Heldwein W, Hoechter W, et al. Much ado about the "ultrashort" Barrett esophagus? Results from the Munich multicenter study. Gastrointest Endosc 2000;51:A114.

14 Nakamura M, Kawano T, Endo M, Iwai T. Intestinal metaplasia at the esophagogastric junction in Japanese patients without clinical Barrett's esophagus. Am J Gastroenterol 1999;94(11):3145-9.

15 Tonelotto EB, Torreaini RS, Coelho NV, Kurban A. Magnifying endoscopy can make the diagnosis of specialized intestinal metaplasia at the esophagogastric junction. Gastrointest Endosc 2000;51:A117.

16 Filipi CJ, Edmundowicz SA, Gostout CJ, Lehman GA, Raijman IL, Rothstein RL, et al. Transoral endoscopic suturing for gastroesophageal reflux disease: A multicenter trial. Gastrointest Endosc 2000;51:A143.

17 Swain P, Park P0, Kjellin T, Gong F, Kadirkamanathan SS, Appleyard M. Endoscopic gastroplasty for gastro-esophageal reflux disease. Gastrointest Endosc 2000;51:A144.

18 Triadafilopoulous G, Utley DS, DiBaise J, Nostrant T, Stollman NH, Rabine J, et al. Radiofrequency energy application to the gastroesophageal junction for the treatment of gastroesophageal reflux disease. Gastrointest Endosc 2000; 51:A223.

19 Roesch T, Helmberger H, Hollerhoff K, Born P, Leban T, Fritz N, et al. Do patients with a low suspicion of biliary and pancreatic disease need MRCP? A prospective study using ERCP as gold standard. Gastrointest Endosc 2000; 51:A188.

20 Fenlon HM, Nunes DP, Schroy PC 3rd, Barish MA, Clarke PD, Ferrucci JT. A comparison of virtual and conventional colonoscopy for the detection of colorectal polyps. N Engl J Med 1999;341(20):1496-503.

21 Mandel JS, Church TR, Ederer F, Bond JH. Colorectal cancer mortality: effectiveness of biennial screening for fecal occult blood. J Nat Canc Inst 1999;91(5):434-7. 
22 Schoenfeld P, Lipscomb S, Crook J, Dominguez J, Butler J, Holmes L, et al. Accuracy of polyp detection by gastroenterologists and nurse endoscopists during flexible sigmoidoscopy: A randomized trial. Gastroenterology 1999; 117:312-8.

23 Winawer SJ, Stewart ET, Zauber AG, Bond JH, Ansel H, Waye JD, et al. A comparison of colonoscopy and doublecontrast barium enema for surveillance after polypectomy. National Polyp Study Working Group. N Engl J Med 2000; 342(24):1766-72.

24 Jarvinen HJ, Aarnio M, Mustonen H, Aktan-Collan K, Aaltonen LA, Peltomaki P, et al. Controlled 15-year trial on screening for colorectal cancer in families with hereditary nonpolyposis colorectal cancer. Gastroenterology 2000; 118(5):829-34.

25 Schatzkin A, Lanza E, Corle D, Lance P, Iber F, Caan B, et al. Lack of effect of a low-fat, high-fiber diet on the recurrence of colorectal adenomas. Polyp Prevention Trial Study Group. N Engl J Med 2000;342(16):1149-55.

26 Alberts DS, Martinez ME, Roe DJ, Guillen-Rodriguez JM, Marshall JR, van Leeuwen JB, et al. Lack of effect of a highfiber cereal supplement on the recurrence of colorectal adenomas. Phoenix Colon Cancer Prevention Physician's Network. N Engl J Med 2000;342(16):1156-62.

27 Sinha R, Chow WH, Kulldorff M, Denobile J, Butler J, Garcia-Closas M, et al. Well-done, grilled red meat increases the risk of colorectal adenomas. Cancer Research 1999; 59(17):4320-4.

28 Baron JA, Beach M, Mandel JS, van Stolk RU, Haile RW, Sandler RS, et al. Calcium supplements for the prevention of colorectal adenomas. N Engl J Med 1999;340(2):101-7.

29 Steinbach G, Lynch PM, Phillips RK, Wallace MH, Hawk E, Gordon GB, et al. The effect of celecoxib, a cyclooxygenase-2 inhibitor, in familial adenomatous polyposis. N Engl J Med 2000;342(26):1946-52.

30 Camilleri M, Northcutt AR, Kong S, Dukes GE, McSorley D, Mangel AW. Efficacy and safety of alosetron in women with irritable bowel syndrome: a randomised, placebocontrolled trial. Lancet 2000;355:1035-40.

31 Bensoussan A, Talley NJ, Hing M, Menzies R, Guo A, Ngu M. Treatment of irritable bowel syndrome with chinese herbal medicine: a randomised controlled trial. JAMA 1998; 280:1585-9.

32 Kahrilas PJ, Shi G, Manka M, Joehl RJ. Increased frequency of transient lower esophageal sphincter relaxation induced by gastric distension in reflux patients with hiatal hernia. Gastroenterology 2000;118(4):688-95.

33 Klinkenberg-Knol EC, Nelis F, Dent J, Snel P, Mitchell B, Prichard P, et al. Long-term omeprazole treatment in resistant gastroesophageal reflux disease: efficacy, safety, and influence on gastric mucosa. Gastroenterology 2000; 118(4):661-9.

34 Lundell L, Miettinen P, Myrvold HE, Pedersen SA, Thor K, Andersson A, et al. Lack of effect of acid suppression therapy on gastric atrophy. Nordic GERD Study Group. Gastroenterology 1999;117(2):319-26.

35 Rudolph RE, Vaughan TL, Storer BE, Haggitt RC, Rabinovitch PS, Levine DS, Reid BJ. Effect of segment length on risk for neoplastic progression in patients with Barrett esophagus. Ann Intern Med 2000;132(8):612-20.

36 Sharma P, Weston AP, Morales T, Topalovski M, Mayo MS, Sampliner RE. Relative risk of dysplasia for patients with intestinal metaplasia in the distal esophagus and in the gastric cardia. Gut 2000;46(1):9-13.

37 Castell D0, Katzka DA. Importance of adequate acid suppression in the management of Barrett's esophagus. Gastroenterology 1999;117(6):1509-10.

38 Ouatu-Lascar R, Fitzgerald RC, Triadafilopoulos G. Differentiation and proliferation in Barrett's esophagus and the effects of acid suppression. Gastroenterology 1999; $117(2): 327-35$.
39 Ortiz A, Martinez de Haro LF, Parrilla P, Molina J, Bermejo J, Munitiz V. 24-h pH monitoring is necessary to assess acid reflux suppression in patients with Barrett's oesophagus undergoing treatment with proton pump inhibitors. Br J Surg 1999;86(11):1472-4

40 Bais JE, Bartelsman JF, Bonjer HJ, Cuesta MA, Go PM, Klinkenberg-Knol EC, et al. Laparoscopic or conventional Nissen fundoplication for gastro-esophageal reflux disease: randomised clinical trial. The Netherlands Antireflux Surgery Study Group. Lancet 2000;355:170-4.

41 Rantanen TK, Salo JA, Sipponen JT. Fatal and life-threatening complications in antireflux surgery: analysis of 5502 operations. Br J Surg 1999;86(12):1573-7.

42 Hui TT, Fass SM, Giurgiu DI, Iida A, Takagi S, Phillips EH. Gastroesophageal disease and nausea: does fundoplication help or hurt? Arch Surg 2000;135(5):545-9.

43 Talley NJ, Axon A, Bytzer P, Holtmann G, Lam SK, Van Zanten S. Management of uninvestigated and functional dyspepsia: a working part report for the World Congress of Gastroenterology 1998. Aliment Pharmacol Ther 1999;13:1135-48.

44 Holtmann G, Cain C, Malfertheiner P. Gastric Helicobacter pylori infection accelerates healing of reflux esophagitis during treatment with proton pump inhibitor pantoprazole. Gastroenterology 1999;117:11-6.

45 El-Serag HB, Sonnenberg A, Jamal MM, Inadomi JM, Crooks LA, Feddersen RM. Corpus gastritis is protective against reflux esophagitis. Gut 1999;45:181-5.

46 0'Connor HJ. Helicobacter pylori and gastroesophageal reflux disease - clinical implications and management. Aliment Pharmacol Ther 1999;13:117-27.

47 Gillen D, Wirz AA, Ardill JE, McColl KE. Rebound hypersecretion after omeprazole and its relation to on-treatment acid suppression and Helicobacter pylori status. Gastroenterology 1999;116:239-47.

48 Weil J, Langman MJ, Wainwright P, Lawson DH, Rawlins M, Logan RF, et al. Peptic ulcer bleeding: accessory risk factors and interactions with non-steroidal anti-inflammatory drugs. Gut 2000;46:27-31.

49 Aalykke C, Lauritsen JM, Hallas J, Reinholdt S, Krogfelt K, Lauritsen K. Helicobacter pylori and risk of ulcer bleeding among users of non-steroidal anti-inflammatory drugs: a case-control study. Gastroenterology 1999;116:1305-9.

50 de Abajo FJ, Rodriguez LA, Montero D. Association between selective serotonin reuptake inhibitors and upper gastrointestinal bleeding: population-based case-control study. Br Med J 1999;319:1106-9.

51 Laine L, Harper S, Simon T, Bath R, Johanson J, Schwartz H, et al. A randomized trial comparing the effect of rofecoxib, a cyclooxygenase 2-specific inhibitor, with that of ibuprofen on the gastroduodenal mucosa of patients with osteoarthritis. Gastroenterology 1999;117:776-83.

52 Feldman M, McMahon AT. Do cyclooxygenase-2-inhibitors provide benefits similar to those of traditional nonsteroidal anti-inflammtory drugs, with less gastrointestinal bleeding? Ann Intern Med 2000;132:134-43.

53 Hayat M, Arora DS, Dixon MF, Clark B, O’Mahony S. Effects of Helicobacter pylori eradication on the natural history of lymphocytic gastritis. Gut 1999;45:495-8.

54 Steinbach G, Ford R, Glober G, Sample D, Hagemeister FB, Lynch PM, et al. Antibiotic treatment of gastric lymphoma of mucosa-associated lymphoid tissue. Ann Intern Med 1999; $131: 88-95$.

55 Annibale B, Marignani M, Monarca B, Antonelli G, Marcheggiano A, Martino G, et al. Reversal of iron deficiency anemia after Helicobacter pylori eradication in patients with asymptomatic gastritis. Ann Intern Med 1999;131:668-72.

56 Unge P. The OAC and OMC options. Eur J Gastroenterol Hepatol 1999;11(Suppl 2):S9-S17. 
57 Lind T, Megraud F, Unge P, Bayerdorffer E, O'morain C, Spiller R, et al. The MACH 2 study: role of omeprazole in eradication of Helicobacter pylori with 1-week triple therapies. Gastroenterology 1999;116:248-53.

58 Tulassay Z, Kryszewski A, Dit P, Kleczkowski D, Rudzinski J, Bartuz Z, et al. 7-day treatment with esomeprazole-based triple therapy eradicates $\mathrm{H}$. pylori $(\mathrm{Hp})$ and heals patients with DU disease. Gastroenterology 2000;118(4):A502.

59 Veldhuyzen Van Zanten S, Lauritsen K, Delchier JC, Labenz J, Martin de Argila C, Lind T, et al. 7-day triple therapy with esomeprazole, amoxicillin and clarithromycin for H. Pylori (Hp) eradication in duodenal ulcer (DU) disease. Gastroenterology 2000;118(4):A503.

60 Furuta T, Shirai N, Takashima M, Xiao F, Hanai H, Ohashi K, Ishizaki T. Effect of genetic differences in CYP2C19 on cure rates for Helicobacter pylori infection by a triple therapy with proton pump inhibitor, amoxicillin, and clarithromycin. Gastroenterology 2000;118(Suppl):2664.

61 De Koster E, Devaster J, Vandenborre C, De Brayne I, Deltenre M. Ten years surveillance of Helicobacter pylori primary resistance to macrolides and imidazoles. Gastroenterology 2000;118(Suppl):2660.

62 De Boer WA, Tytgat GN. Treatment of Helicobacter pylori infection. Br Med J 2000;320:31-4.

63 Adamsson I, Nord CE, Lundquist P, Sjostedt S, Edlund C. Comparative effects of omeprazole, amoxicillin plus metronidazole, versus omeprazole, clarithromycin plus metronidazole on the oral, gastric and intestinal microflora in Helicobacter pylori-infected patients. J Antimicrob Chemother 1999;44:629-40.

64 Razavi B. Reactive arthritis after Helicobacter pylori eradication. Lancet 2000;355:720.

65 Stack WA, Mann SD, Roy AJ, Heath P, Sopwith M, Freeman J, et al. Randomised controlled trial of CDP571 antibody to tumour necrosis factor-alpha in Crohn's disease. Lancet 1997;349:521-4.

66 Targan SR, Hanauer SB, van Deventer SJ, Mayer L, Present DH, Braakman T, et al. A short-term study of chimeric monoclonal antibody cA2 to tumor necrosis factor alpha for Crohn's disease. Crohn's Disease cA2 Study Group. N Engl J Med 1997;337:1029-35.

67 Present DH, Rutgeerts P, Targan S, Hanauer SB, Mayer L, van Hogezand RA, et al. Infliximab for the treatment of fistulas in patients with Crohn's disease. N Engl J Med 1999; 340:1398-405.
68 Rutgeerts P, D’Haens G, Targan S, Vasiliauskas E, Hanauer SB, Present DH, et al. Efficacy and safety of retreatment with anti-tumor necrosis factor antibody (infliximab) to maintain remission in Crohn's disease. Gastroenterology 1999; 117:761-9.

69 Asten P, Barrett J, Symmons D. Risk of developing certain malignancies is related to duration of immunosuppressive drug exposure in patients with rheumatic diseases. J Rheumatol 1999;26:1705-14.

70 Bickston SJ, Lichtenstein GR, Arseneau K0, Cohen RB, Cominelli F. The relationship between infliximab treatment and lymphoma in Crohn's disease. Gastroenterology 1999; 117:1433-7.

71 Neurath MF, Wanitschke R, Peters M, Krummenauer F, Meyer zum Buschenfelde KH, Schlaak JF. Randomised trial of mycophenolate mofetil versus azathioprine for treatment of chronic active Crohn's disease. Gut 1999;44:625-8.

72 Fellermann K, Steffen M, Stein J, Raedler A, Hamling J, Ludwig D, et al. Mycophenolate mofetil: lack of efficacy in chronic active inflammatory bowel disease. Aliment Pharmacol Ther 2000;14:171-6.

73 Feagan BG, Fedorak RN, Irvine EJ, Wild G, Sutherland L, Steinhart AH, et al. A comparison of methotrexate with placebo for the maintenance of remission in Crohn's disease. North American Crohn's Study Group Investigators. N Engl J Med 2000;342:1627-32.

74 Slonim AE, Bulone L, Ritz S, Goldberg T, Chen A, Martiniuk F. Identification of two subtypes of infantile acid maltase deficiency. J Pediatr 2000;137(2):283-5.

75 Lochs H, Mayer M, Fleig WE, Mortensen PB, Bauer P, Genser D, et al. Prophylaxis of postoperative relapse in Crohn's disease with mesalamine: European Cooperative Crohn's Disease Study VI. Gastroenterology 2000;118:264-73.

76 Rembacken BJ, Snelling AM, Hawkey PM, Chalmers DM, Axon AT. Non-pathogenic Escherichia coli versus mesalazine for the treatment of ulcerative colitis: a randomised trial. Lancet 1999;354:635-9.

77 Vestergaard P, Krogh K, Rejnmark L, Laurberg S, Mosekilde L. Fracture risk is increased in Crohn's disease, but not in ulcerative colitis. Gut 2000;46:176-81.

78 Silverstein MD, Loftus EV, Sandborn WJ, Tremaine WJ, Feagan BG, Nietert PJ, et al. Clinical course and costs of care for Crohn's disease: Markov model analysis of a populationbased cohort. Gastroenterology 1999;117:49-57. 